PROCEEDINGS OF THE

AMERICAN MATHEMATICAL SOCIETY

Volume 139, Number 6, June 2011, Pages 2207-2215

S 0002-9939(2010)10649-4

Article electronically published on November 29, 2010

\title{
SOME GEOMETRIC PROPERTIES OF HYPERSURFACES WITH CONSTANT $r$-MEAN CURVATURE IN EUCLIDEAN SPACE
}

\author{
DEBORA IMPERA, LUCIANO MARI, AND MARCO RIGOLI
}

(Communicated by Jianguo Cao)

\begin{abstract}
Let $f: M \rightarrow \mathbb{R}^{m+1}$ be an isometrically immersed hypersurface. In this paper, we exploit recent results due to the authors to analyze the stability of the differential operator $L_{r}$ associated with the $r$ th Newton tensor of $f$. This appears in the Jacobi operator for the variational problem of minimizing the $r$-mean curvature $H_{r}$. Two natural applications are found. The first one ensures that under a mild condition on the integral of $H_{r}$ over geodesic spheres, the Gauss map meets each equator of $\mathbb{S}^{m}$ infinitely many times. The second one deals with hypersurfaces with zero $(r+1)$-mean curvature. Under similar growth assumptions, we prove that the affine tangent spaces $f_{*} T_{p} M, p \in M$, fill the whole $\mathbb{R}^{m+1}$
\end{abstract}

\section{INTRODUCTION}

In what follows, $f: M^{m} \rightarrow \mathbb{R}^{m+1}$ will always denote a connected, orientable, complete, noncompact hypersurface of Euclidean space. We fix an origin $o \in M$ and let $r(x)=\operatorname{dist}(x, o), x \in M$. We set $B_{r}$ and $\partial B_{r}$ for, respectively, the geodesic ball and the geodesic sphere centered at $o$ with radius $r$. Moreover, let $\nu$ be the spherical Gauss map and denote with $A$ both the second fundamental form and the shape operator in the orientation of $\nu$. Associated with $A$ we have the principal curvatures $k_{1}, \ldots, k_{m}$ and the set of symmetric functions $S_{j}$ :

$$
S_{j}=\sum_{i_{1}<i_{2}<\ldots<i_{j}} k_{i_{1}} \cdot k_{i_{2}} \cdot \ldots \cdot k_{i_{j}}, \quad j \in\{1, \ldots, m\}, \quad S_{0}=1 .
$$

The $j$-mean curvature of $f$ is defined as

$$
H_{0}=1, \quad\left(\begin{array}{l}
n \\
j
\end{array}\right) H_{j}=S_{j},
$$

so that, for instance, $H_{1}$ is the mean curvature and $H_{m}$ is the Gauss-Kronecker curvature of the hypersurface. Note that, when changing the orientation $\nu$, the odd curvatures change sign, while the sign of the even curvatures is an invariant of the immersion. By the Gauss equations and the flatness of $\mathbb{R}^{m+1}$ it is easy to see that

$$
H_{2}=\left(\begin{array}{c}
m \\
2
\end{array}\right)^{-1} S_{2}=\frac{1}{2}\left(\begin{array}{c}
m \\
2
\end{array}\right)^{-1} \text { scal }
$$

Received by the editors March 31, 2010 and, in revised form, June 14, 2010

2010 Mathematics Subject Classification. Primary 53C21, 53C42; Secondary 58J50, 53A10. 
where scal is the scalar curvature of $M$. The $j$-mean curvatures satisfy the so-called Newton inequalities

$$
H_{j}^{2} \geq H_{j-1} H_{j+1},
$$

equality holding if and only if $p$ is an umbilical point (see [9]). We stress that no restriction is made on the sign of the $H_{i}$ 's.

Theorem 1.1. Let $f: M \rightarrow \mathbb{R}^{m+1}$ be a hypersurface such that, for some $j \in$ $\{0, m-2\}, H_{j+1}$ is a nonzero constant. If $j \geq 1$, assume that there exists a point $p \in M$ at which the second fundamental form is definite. Set

$$
v_{j}(r)=\int_{\partial B_{r}} H_{j}, \quad v_{1}(r)=\int_{\partial B_{r}} H_{1}
$$

where integration is with respect to the $(m-1)$-dimensional Hausdorff measure of $\partial B_{r}$. Fix an equator $E \subset \mathbb{S}^{m}$ and suppose that either

$$
\begin{aligned}
& \text { (i) } \int^{+\infty} \frac{\mathrm{d} r}{v_{j}(r)}=+\infty \quad \text { and } \quad H_{1} \notin L^{1}(M) \quad \text { or } \\
& \text { (ii) } \int^{+\infty} \frac{\mathrm{d} r}{v_{j}(r)}<+\infty \quad \text { and } \\
& \quad \limsup _{r \rightarrow+\infty} \sqrt{v_{1}(r) v_{j}(r)} \int_{r}^{+\infty} \frac{\mathrm{d} s}{v_{j}(s)}>\frac{1}{2}\left[(j+1)\left(\begin{array}{c}
m+1 \\
j+2
\end{array}\right) H_{j+1}\right]^{-1 / 2} .
\end{aligned}
$$

Then, there exists a divergent sequence $\left\{x_{k}\right\} \subset M$ such that $\nu\left(x_{k}\right) \in E$, where $\nu$ is the spherical Gauss map.

Remark 1.2. Up to changing the orientation of $M$, we can suppose that the second fundamental form at $p$ is positive definite. As we will see later in more detail, this has the remarkable consequence that each $H_{i}, 1 \leq i \leq n$, is strictly positive at every point of $M$. In particular, $v_{1}$ and $v_{j}$ are both strictly positive and the requirements in (2) are meaningful.

Remark 1.3. When $j=1$, the existence of an elliptic point $p \in M$ can be replaced by requiring $\mathrm{H}_{2}$ to be a positive constant; see [6] for details. The case $j=0$ has been considered in [4].

We clarify the role of $(i)$ and $(i i)$ with some examples. First, we deal with the case $j \neq 1$, and we assume that $v_{j}$ is of order $r^{k}\left(\operatorname{resp} e^{k r}\right)$, for some $k>0$. Then assumption (ii) requires that $v_{1}(r)$ is of order at least $r^{k-2}\left(\operatorname{resp} e^{k r}\right)$. Roughly speaking, $v_{1}$ has to be big enough with respect to the other integral curvature $v_{j}$. Under additional requirements on the intrinsic curvatures of $M$, standard volume comparisons allow us to control the volume of $\partial B_{r}$ and (ii) can be read as $H_{1}$ not decaying too fast at infinity. When $j=1$, things are somewhat different. Indeed, (ii) implies that $v_{1}(r)$ does not grow too fast; that is, loosely speaking, it has at most exponential growth. This shows that two opposite effects balance in condition (ii). The same happens for $(i)$ with $j=1$ as a consequence of the Cauchy-Schwarz inequality and the coarea formula

$$
\left(\int_{R}^{r} \frac{\mathrm{d} s}{v_{1}(s)}\right)\left(\int_{B_{r} \backslash B_{R}} H_{1}\right) \geq(r-R)^{2} .
$$


Finally, we stress that $(i)$ and $(i i)$ are mild hypotheses as they only involve the integral of extrinsic curvatures. In other words, no pointwise control is required.

Up to identifying the image of the tangent space at $p \in M$ with an affine hyperplane of $\mathbb{R}^{m+1}$ in the standard way, we can also prove the following result:

Theorem 1.4. Let $f: M \rightarrow \mathbb{R}^{m+1}$ be a hypersurface with $H_{j+1} \equiv 0$. If $j \geq$ 1 , assume $\operatorname{rank}(A)>j$ at every point. Define $v_{1}, v_{j}$ as in (11). Then, under assumptions (2) ( $i)$ or (ii), for every compact set $K \subset M$ we have

$$
\bigcup_{p \in M \backslash K} T_{p} M \equiv \mathbb{R}^{m+1}
$$

that is, the tangent envelope of $M \backslash K$ coincides with $\mathbb{R}^{m+1}$.

Remark 1.5. As we will see later, condition $\operatorname{rank}(A)>j$ implies that $H_{i}>0$ for every $1 \leq i \leq j$.

\section{Preliminaries}

We start by recalling the definition and some properties of the Newton tensors $P_{j}, j \in\{0, \ldots, m\}$. They are inductively defined by

$$
P_{0}=I, \quad P_{j}=S_{j} I-A P_{j-1} .
$$

For future use, we state the following algebraic lemma. For a proof, see 3 .

Lemma 2.1. Let $\left\{e_{i}\right\}$ be the principal directions associated with $A, A e_{i}=k_{i} e_{i}$, and let $S_{j}\left(A_{i}\right)$ be the $j$-th symmetric function of $A$ restricted to the $(m-1)$-dimensional space $e_{i}^{\perp}$. Then, for each $1 \leq j \leq m-1$,

$$
\begin{aligned}
& \text { (1) } A P_{j}=P_{j} A \\
& \text { (2) } P_{j} e_{i}=S_{j}\left(A_{i}\right) e_{i} ; \\
& \text { (3) } \operatorname{Tr}\left(P_{j}\right)=\sum_{i} S_{j}\left(A_{i}\right)=(m-j) S_{j} ; \\
& \text { (4) } \operatorname{Tr}\left(A P_{j}\right)=\sum_{i} k_{i} S_{j}\left(A_{i}\right)=(j+1) S_{j+1} ; \\
& \text { (5) } \operatorname{Tr}\left(A^{2} P_{j}\right)=\sum_{i} k_{i}^{2} S_{j}\left(A_{i}\right)=S_{1} S_{j+1}-(j+2) S_{j+2} .
\end{aligned}
$$

It follows from (2) in the above lemma and from the definition of $P_{m}$ that $P_{m}=0$. Related to the $j$-th Newton tensor there is a well-defined, symmetric differential operator acting on $C_{c}^{\infty}(M)$ :

$$
L_{j} u=\operatorname{Tr}\left(P_{j} \operatorname{Hess}(u)\right)=\operatorname{div}\left(P_{j} \nabla u\right) \quad \forall u \in C_{c}^{\infty}(M),
$$

where the last equality is due to the fact that $A$ is a Codazzi tensor in $\mathbb{R}^{m+1}$; see [5], [13]. $L_{j}$ naturally appears when looking for stationary points of the curvature integral

$$
\mathcal{A}_{j}(M)=\int_{M} S_{j} \mathrm{~d} V_{M}
$$

for compactly supported volume-preserving variations. These functionals can be viewed as a generalization of the volume functional. In fact, in [3] and 6 the stationary points of $\mathcal{A}_{j}$ are characterized as those immersions having constant $S_{j+1}$. In the above-mentioned paper [6], M.F. Elbert computes the second variation of $\mathcal{A}_{j}$ in more general ambient spaces and obtains in the Euclidean setting the expression

$$
T_{j}=L_{j}+\left(S_{1} S_{j+1}-(j+2) S_{j+2}\right)
$$


for the Jacobi operator. In what follows we are interested in the case of $L_{j}$ elliptic. There are a number of different results giving sufficient conditions to guarantee this fact, and the next two fit the situation of our main theorems.

Proposition 2.2. Let $M$ be an m-dimensional connected, orientable hypersurface of some space form $N$. Then, $L_{i}$ is elliptic for every $1 \leq i \leq j$ in each of the following cases:

(i) $M$ contains an elliptic point, that is, a point $p \in M$ at which $A$ is definite (positive or negative), and $S_{j+1} \neq 0$ at every point of $M$. Note that up to changing the orientation of $M$, we can assume $A_{p}$ to be positive definite, and by continuity $S_{j+1}>0$ on $M$.

(ii) $S_{j+1} \equiv 0$ and $\operatorname{rank}(A)>j$ at every point of $M$.

Moreover, in both cases, every $i$-mean curvature $H_{i}$ is strictly positive on $M$, for $1 \leq i \leq j$.

For a proof of $(i)$, see [3], while for $(i i)$, see [10].

From the above proposition, the requirements on $p$ and $\operatorname{rank}(A)$ in the main theorems ensure ellipticity. As stressed in Remark 1.3, when $j=2$ in [6] it is shown that the sole requirement $H_{2}>0$ implies the ellipticity of $L_{1}$. In the assumptions of the above proposition, we can define the $j$-volume of some measurable subset $K \subset M$ as the integral

$$
\mathcal{A}_{j}(K)=\int_{K} S_{j} \mathrm{~d} V_{M}
$$

Hereafter, we restrict ourselves to the case that $L_{j}$ is elliptic. Given the relatively compact domain $\Omega \subset M, L_{j}$ is bounded from below on $C_{c}^{\infty}(\Omega)$ and, by Rellich's theorem, for a sufficiently large $\lambda,\left(L_{j}-\lambda\right)$ is invertible with compact resolvent. By standard spectral theory, $L_{j}$ is therefore essentially self-adjoint on $C_{c}^{\infty}(\Omega)$ (Theorem 3.3.2 in [12]). Essential self-adjointness implies that $C_{c}^{\infty}(\Omega)$ and $\operatorname{Lip}_{0}(\Omega)$ are cores for the quadratic form associated to $L_{j}$. The first eigenvalue $\lambda_{1}^{T_{j}}(\Omega)$, with Dirichlet boundary condition, is therefore defined by the Rayleigh characterization

$$
\lambda_{1}^{T_{j}}(\Omega)=\inf _{\substack{\phi \in \operatorname{Lip}_{0}(\Omega) \\ \phi \neq 0}} \frac{\int_{\Omega}\left\langle P_{j}(\nabla \phi), \nabla \phi\right\rangle-\int_{\Omega}\left(S_{1} S_{j+1}-(j+2) S_{j+2}\right) \phi^{2}}{\int_{\Omega} \phi^{2}},
$$

where $\operatorname{Lip}_{0}(\Omega)$ can be replaced with $C_{c}^{\infty}(\Omega)$. By the monotonicity property of eigenvalues (or, in other words, since $L_{j}$ satisfies the unique continuation property, [2]), if $\Omega_{1}$ is a domain with compact closure in $\Omega_{2}$, and $\Omega_{2} \backslash \Omega_{1}$ has nonempty interior, then $\lambda_{1}^{T_{j}}\left(\Omega_{1}\right)>\lambda_{1}^{T_{j}}\left(\Omega_{2}\right)$. Hence, we deduce the existence of

$$
\lambda_{1}^{T_{j}}(M)=\lim _{\mu \rightarrow+\infty} \lambda_{1}^{T_{j}}\left(\Omega_{\mu}\right)
$$

where $\left\{\Omega_{\mu}\right\}$ is any exhaustion of $M$ by means of increasing, relatively compact domains with smooth boundary. The next result is substantially an application of the result of Moss-Piepenbrink [11, slightly modified according to Fischer-Colbrie and Schoen [8] and Fischer-Colbrie [7] (consult also [12], Chapter 3, and for the case of $\left.L_{1}, 6\right]$ ).

Proposition 2.3. Let $M$ be a Riemannian manifold and let $T_{j}$ be as above. The following statements are equivalent:

(i) $\lambda_{1}^{T_{j}}(M) \geq 0$; 
(ii) there exists $u \in C^{\infty}(M), u>0$, that is a solution of $T_{j} u=0$ on $M$.

Furthermore, there exists a compact set $K \subset M$ and $u \in C^{\infty}(M \backslash K), u>0$, that is a solution of $T_{j} u=0$ on $M \backslash K$ if and only if $\lambda_{1}^{T_{j}}(M \backslash K) \geq 0$.

Next, we need to consider the following Cauchy problem (here, as usual, $\mathbb{R}^{+}=$ $(0,+\infty)$ and $\left.\mathbb{R}_{0}^{+}=[0,+\infty)\right)$ :

$$
\left\{\begin{array}{l}
\left(v(t) z^{\prime}(t)\right)^{\prime}+A(t) v(t) z(t)=0 \quad \text { on } \mathbb{R}^{+}, \\
z^{\prime}(t)=\mathrm{O}(1) \text { as } t \downarrow 0^{+}, \quad z\left(0^{+}\right)=z_{0}>0,
\end{array}\right.
$$

where $A(t)$ and $v(t)$ satisfy the following conditions:

(A1) $A(t) \in L_{\text {loc }}^{\infty}\left(\mathbb{R}_{0}^{+}\right), \quad A(t) \geq 0, \quad A \not \equiv 0$ in the $\mathrm{L}_{\text {loc }}^{\infty}$ sense;

(V1) $v(t) \in L_{\mathrm{loc}}^{\infty}\left(\mathbb{R}_{0}^{+}\right), v(t) \geq 0, \frac{1}{v(t)} \in L_{\mathrm{loc}}^{\infty}\left(\mathbb{R}^{+}\right)$;

(V2) there exists $a \in \mathbb{R}^{+}$such that $v$ is increasing on $(0, a)$ and $\lim _{t \rightarrow 0^{+}} v(t)=0$.

Observe that (V2) has to be interpreted as there exists a version of $v$ which is increasing near 0 and whose limit as $t \rightarrow 0^{+}$is 0 .

By Proposition $A .1$ of [4, under the above assumptions (44) has a solution $z(t) \in$ $\operatorname{Lip}_{\text {loc }}\left(\mathbb{R}_{0}^{+}\right)$(and the condition $z^{\prime}(t)=\mathrm{O}(1)$ as $t \downarrow 0^{+}$is satisfied in an appropriate sense). Furthermore by Proposition $A .3$ of [4], $z(t)$ has only isolated zeros. In the case that $1 / v \in L^{1}((1,+\infty))$, by Proposition 2.5 of [4] if, for some $T>0$,

$$
\limsup _{t \rightarrow \infty} \frac{\int_{T}^{t} \sqrt{A(s)} \mathrm{d} s}{-\frac{1}{2} \log \int_{t}^{+\infty} \frac{\mathrm{d} s}{v(s)}}>1,
$$

then every solution of

$$
\left\{\begin{array}{l}
\left(v(t) z^{\prime}(t)\right)^{\prime}+A(t) v(t) z(t)=0 \quad \text { on }\left(t_{0},+\infty\right), t_{0}>0 \\
z\left(t_{0}\right)=z_{0}>0
\end{array}\right.
$$

has isolated zeros and is oscillatory. The same happens if

$$
\int^{+\infty} \frac{\mathrm{d} t}{v(t)}=+\infty \quad \text { and } \quad \int^{+\infty} A(t) v(t) \mathrm{d} t=+\infty
$$

(see Corollary 2.4 of [4]).

A final result that we shall use is the following computation. (For a proof, see [13] , 1.)

Proposition 2.4. Let $f: M \rightarrow \mathbb{R}^{m+1}$ be an isometric immersion of an oriented hypersurface and $\nu: M \rightarrow \mathbb{S}^{m}$ its Gauss map. Fix $a \in \mathbb{S}^{m}$. Then

$$
\begin{aligned}
& L_{j}\langle a, \nu\rangle=-\left(S_{1} S_{j+1}-(j+2) S_{j+2}\right)\langle a, \nu\rangle-\left\langle\nabla S_{j+1}, a\right\rangle, \\
& L_{j}\langle f, \nu\rangle=-(j+1) S_{j+1}-\left(S_{1} S_{j+1}-(j+2) S_{j+2}\right)\langle f, \nu\rangle-\left\langle\nabla S_{j+1}, f\right\rangle,
\end{aligned}
$$

where $\langle$,$\rangle stands for the scalar product of vectors in \mathbb{S}^{m} \subset \mathbb{R}^{m+1}$.

In particular, if $S_{j+1}$ is constant, we have $T_{j}\langle a, \nu\rangle=0$. Moreover, if $S_{j+1} \equiv 0$, then $T_{j}\langle f, \nu\rangle \equiv 0$. 


\section{Proof of Theorem 1.1}

Fix an equator $E$ and reason by contradiction: assume that there exists a sufficiently large geodesic ball $B_{R}$ such that, outside $B_{R}, \nu$ does not meet $E$. In other words, $\nu\left(M \backslash B_{R}\right)$ is contained in the open spherical caps determined by $E$. Indicating with $a \in \mathbb{S}^{m}$ one of the two focal points of $E,\langle a, \nu(x)\rangle \neq 0$ on $M \backslash B_{R}$.

Let $\mathcal{C}$ be one of the (finitely many) connected components of $M \backslash B_{R}$; then $\nu(\mathcal{C})$ is contained in only one of the open spherical caps determined by $E$. Up to replacing $a$ with $-a$, we can suppose $u=\langle a, \nu\rangle>0$ on $\mathcal{C}$. Proceeding in the same way for each connected component, we can construct a positive function $u$ on $M \backslash B_{R}$. Since $S_{j+1}$ is constant, by Proposition 2.4 we have that $u>0$ satisfies

$$
T_{j} u=L_{j} u+\left(S_{1} S_{j+1}-(j+2) S_{j+2}\right) u=0
$$

on $M \backslash B_{R}$. Thus, by Proposition 2.3 $\lambda_{1}^{T_{j}}\left(M \backslash B_{R}\right) \geq 0$. We shall now show that the assumptions of the theorem contradict this fact. As already stressed, the existence of an elliptic point forces both $H_{j}$ and $H_{j+1}$ to be positive. Fix a radius $0<R_{0}<R$ and let $K_{j}$ be a smooth positive function on $M$ such that

$$
K_{j}(x)=\left\{\begin{array}{ll}
1 & \text { on } B_{R_{0} / 2}, \\
(m-j) S_{j} & \text { on } M \backslash B_{R_{0}}
\end{array} .\right.
$$

Next, we define

$$
v_{j}(t)=\int_{\partial B_{t}} K_{j}
$$

Using Proposition 1.2 of 4 ] we see that $v_{j}(t)$ satisfies $(\mathrm{V} 1)$ with $v_{j}(t)>0$ on $\mathbb{R}^{+}$ and (V2). Next, we define

$$
A(t)=\frac{1}{v_{j}(t)} \int_{\partial B_{t}} S_{1} S_{j+1}-(j+2) S_{j+2} .
$$

Then, repeated applications of Newton's inequalities give

$$
H_{1} H_{j+1}-H_{j+2} \geq 0 \text {. }
$$

Thus, using (12),

$$
\begin{aligned}
& S_{1} S_{j+1}-(j+2) S_{j+2}=m\left(\begin{array}{c}
m \\
j+1
\end{array}\right) H_{1} H_{j+1}-(j+2)\left(\begin{array}{c}
m \\
j+2
\end{array}\right) H_{j+2} \\
& =\left(\begin{array}{c}
m \\
j+1
\end{array}\right)\left(m H_{1} H_{j+1}-(m-j-1) H_{j+2}\right) \\
& \geq\left(\begin{array}{c}
m \\
j+1
\end{array}\right)\left[m-\frac{m-j-1}{j+2}\right] H_{1} H_{j+1}=(j+1)\left(\begin{array}{c}
m+1 \\
j+2
\end{array}\right) H_{1} H_{j+1} \geq 0 .
\end{aligned}
$$

This implies that $A(t) \geq 0$, and

$$
A(t) v_{j}(t) \geq(j+1)\left(\begin{array}{c}
m+1 \\
j+2
\end{array}\right) H_{j+1} \int_{\partial B_{t}} H_{1}=(j+1)\left(\begin{array}{c}
m+1 \\
j+2
\end{array}\right) H_{j+1} v_{1}(t) .
$$

If $1 / v_{j} \notin L^{1}((1,+\infty))$, then under (2) $(i)$ and by the coarea formula we deduce that $A v_{j} \notin L^{1}\left(\mathbb{R}^{+}\right)$. Hence, we can apply (7) to deduce that every solution of

$$
\left\{\begin{array}{l}
\left(v_{j}(t) z^{\prime}(t)\right)^{\prime}+A(t) v_{j}(t) z(t)=0 \quad \text { on }\left(t_{0},+\infty\right), t_{0}>0 \\
z\left(t_{0}\right)=z_{0}>0
\end{array}\right.
$$


is oscillatory. The same conclusion holds when $1 / v_{j} \in L^{1}((1,+\infty))$. Indeed, from (11), (13),

$$
\frac{\int_{T}^{t} \sqrt{A(s)} \mathrm{d} s}{-\frac{1}{2} \log \int_{t}^{+\infty} \frac{\mathrm{d} s}{v_{j}(s)}} \geq 2 \sqrt{(j+1)\left(\begin{array}{c}
m+1 \\
j+2
\end{array}\right) H_{j+1}} \frac{\int_{T}^{t} \sqrt{\frac{v_{1}(s)}{v_{j}(s)}} \mathrm{d} s}{-\log \int_{t}^{+\infty} \frac{\mathrm{d} s}{v_{j}(s)}} .
$$

Using de l'Hôpital's rule and (2) (ii), (51) is met. Now let $R<T_{1}<T_{2}$ be two consecutive zeros of $z(t)$ after $R$. Define

$$
\psi(x)= \begin{cases}z(r(x)) & \text { on } \overline{B_{T_{2}}} \backslash B_{T_{1}} \\ 0 & \text { outside } \overline{B_{T_{2}}} \backslash B_{T_{1}} .\end{cases}
$$

Note that $\psi \equiv 0$ on $\partial\left(\overline{B_{T_{2}}} \backslash B_{T_{1}}\right), \psi \in \operatorname{Lip}_{0}(M)$ and $\nabla \psi(x)=z^{\prime}(r(x)) \nabla r(x)$ where defined. Furthermore, by the coarea formula and the definition of $A(t)$ we have

$$
\begin{array}{r}
\int_{M}\left(S_{1} S_{j+1}-(j+2) S_{j+2}\right) \psi^{2}=\int_{T_{1}}^{T_{2}} z^{2}(t) \int_{\partial B_{t}}\left(S_{1} S_{j+1}-(j+2) S_{j+2}\right) \mathrm{d} t \\
=\int_{T_{1}}^{T_{2}} z^{2}(t) A(t) v_{j}(t) \mathrm{d} t=(m-j) \int_{M} S_{j} A(r) \psi^{2} .
\end{array}
$$

Thus, using (4), the above identity and again the coarea formula,

$$
\begin{aligned}
& \int_{M}\left\langle P_{j}(\nabla \psi), \nabla \psi\right\rangle-\left(S_{1} S_{j+1}-(j+2) S_{j+2}\right) \psi^{2} \\
& \leq \int_{M} \operatorname{Tr}\left(P_{j}\right)|\nabla \psi|^{2}-\left(S_{1} S_{j+1}-(j+2) S_{j+2}\right) \psi^{2} \\
& =\int_{M}(m-j) S_{j}|\nabla \psi|^{2}-\left(S_{1} S_{j+1}-(j+2) S_{j+2}\right) \psi^{2} \\
& =(m-j) \int_{\overline{B_{T_{2}}} \backslash B_{T_{1}}} S_{j}\left[\left(z^{\prime}\right)^{2}-A(t) z^{2}\right] \\
& =(m-j) \int_{T_{1}}^{T_{2}}\left[\left(z^{\prime}\right)^{2}-A(t) z^{2}\right] v_{j}(t) \mathrm{d} t \\
& =(m-j)\left\{\left.z(t) z^{\prime}(t) v_{j}(t)\right|_{T_{1}} ^{T_{2}}-\int_{T_{1}}^{T_{2}}\left[\left(v_{j}(t) z^{\prime}(t)\right)^{\prime}+A(t) v_{j}(t) z(t)\right] z(t) \mathrm{d} t=0 .\right.
\end{aligned}
$$

It follows that

$$
\lambda_{1}^{T_{j}}\left(\overline{B_{T_{2}}} \backslash B_{T_{1}}\right) \leq \frac{1}{\int_{M} \psi^{2}}\left\{\int_{M}\left\langle P_{j}(\nabla \psi), \nabla \psi\right\rangle-\left(S_{1} S_{j+1}-(j+2) S_{j+2}\right) \psi^{2}\right\}=0 .
$$

As a consequence, $\lambda_{1}^{T_{j}}\left(M \backslash B_{R}\right)<0$, which gives the desired contradiction. 
Remark 3.1. As a matter of fact, the orientability of $M$ is not needed. If $M$ is nonorientable, $\nu$ is not globally defined. However, changing the sign of $\nu$ does not change either the assumptions or the conclusion of Theorem 1.1, since the antipodal map on $\mathbb{S}^{m}$ leaves each $E$ fixed. If $\langle a, \nu\rangle \neq 0$ on $M \backslash B_{R}$, the normal field $X=\langle a, \nu\rangle \nu$ is nowhere vanishing and globally defined on $M \backslash B_{R}$. This shows that, in any case, every connected component of $M \backslash B_{R}$ is orientable.

\section{Proof of Theorem 1.4}

Assume that for some $K$ the tangent envelope of $M \backslash K$ does not coincide with $\mathbb{R}^{m+1}$. By choosing Cartesian coordinates appropriately, we can assume that

$$
0 \notin \bigcup_{p \in M \backslash K} T_{p} M
$$

Then the function $u=\langle f, \nu\rangle$ is nowhere vanishing and smooth on $M \backslash K$. Up to changing the orientation, $u>0$ on $M \backslash K$. By Proposition 2.4 $T_{j} u=-(j+$ 1) $S_{j+1}=0$. Note that here the assumption $H_{j+1} \equiv 0$ is essential. It follows that $\lambda_{1}^{T_{j}}(M \backslash K) \geq 0$. The rest of the proof is identical to that of Theorem 1.1. Again, according to Remark 3.1 we can drop the orientability assumption on $M$. Indeed, if the tangent envelope of $M \backslash K$ does not cover $\mathbb{R}^{m+1}$, the vector field $X=\langle f, \nu\rangle \nu$ is a globally defined, nowhere vanishing normal vector field on $M \backslash K$; hence $M \backslash K$ is orientable.

\section{REFERENCES}

1. H. Alencar and A. G. Colares, Integral formulas for the $r$-mean curvature linearized operator of a hypersurface., Ann. Global Anal. Geom. 16 (1998), 203-220. MR1626663 (99k:53075)

2. N. Aronszajn, A unique continuation theorem for solutions of elliptic partial differential equations or inequalities of second order, J. Math. Pures Appl. 36 (1957), 235-249. MR0092067 $(19: 1056 \mathrm{c})$

3. J. L. M. Barbosa and A. G. Colares, Stability of hypersurfaces with constant $r$-mean curvature, Ann. Glob. Anal. Geom. 15 (1997), 277-297. MR1456513 (98h:53091)

4. B. Bianchini, L. Mari, and M. Rigoli, Spectral radius, index estimates for Schrödinger operators and geometric applications, Journ. Funct. Anal. 256 (2009), 1769-1820. MR.2498559 (2010a:58038)

5. S. Y. Cheng and S. T. Yau, Hypersurfaces with constant scalar curvature, Math. Ann. 225 (1977), 195-204. MR0431043 (55:4045)

6. M. F. Elbert, Constant positive 2-mean curvature hypersurfaces, Ill. J. Math. 46 (2002), 247-267. MR1936088 (2003g:53103)

7. D. Fisher-Colbrie, On complete minimal surfaces with finite Morse index in three manifolds, Invent. Math. 82 (1985), 121-132. MR808112 (87b:53090)

8. D. Fisher-Colbrie and R. Schoen, The structure of complete stable minimal surfaces in 3manifolds of nonnegative scalar curvature, Comm. Pure Appl. Math. XXXIII (1980), 199211. MR0562550(81i:53044)

9. G. H. Hardy, J. E. Littlewood, and G. Polya, Inequalities (2nd ed.), Cambridge University Press, 1952. MR0046395(13:727e)

10. J. Hounie and M. L. Leite, The maximum principle for hypersurfaces with vanishing curvature functions, J. Differential Geom. 41 (1995), 247-258. MR1331967 (96b:53080)

11. W. F. Moss and J. Piepenbrink, Positive solutions of elliptic equations, Pac. J. Math. 75 (1978), 219-226. MR500041 (80b:35008)

12. S. Pigola, M. Rigoli, and A. G. Setti, Vanishing and finiteness results in geometric analysis. A generalization of the Bochner technique, Progress in Mathematics, vol. 266, Birkhäuser Verlag, Basel, 2008. MR 2401291 (2009m:58001)

13. H. Rosenberg, Hypersurfaces of constant curvature in space forms, Bull. Sc. Math., $2^{e}$ Série 117 (1993), 211-239. MR1216008 (94b:53097) 
Dipartimento di Matematica, Università degli Studi di Milano, Via Saldini 50, I-20133 Milano, Italy

E-mail address: debora.impera@unimi.it

Dipartimento di Matematica, Università degli Studi di Milano, Via Saldini 50, I-20133 Milano, Italy

E-mail address: luciano.mari@unimi.it

Dipartimento di Matematica, Università degli Studi di Milano, Via Saldini 50, I-20133 Milano, Italy

E-mail address: marco.rigoli@unimi.it 